\title{
Theory of directed localization in one dimension
}

\author{
P W Brouwer, P G Silvestrov, , and C W J Beenakker \\ Instltuut Lorentz, Letden University PO Box 9506, 2300 RA Lelden, The Netherlands
}

(Recelved 19 May 1997)

\begin{abstract}
We present an analytical solution of the delocalization transition that is induced by an imaginary vector potential in a disoidered chain [N Hatano and D R Nelson, Phys Rev Lett 77, 570 (1996)] We compute the ielation between the real and imaginary parts of the energy in the thermodynamic limit, as well as finite-size effects The results are in good agreement with numerical simulations for weak disorder (in which the mean free path is laige compared to the wavelength) [S0163-1829(97)51032 0]
\end{abstract}

In a recent paper, ${ }^{\prime}$ Hatano and Nelson have demonstrated the existence of a mobility edge in a disordered ring with an imaginary vector potentıal A non-Hermitian Hamıltonıan containing an imaginary vector potential arises from the study of the pinning of vortices by columnar defects in a superconducting cylinder ${ }^{2}$ Their discovery of a delocalization transition in one- and two-dimensional systems has generated considerable interest, ${ }^{3}{ }^{5}$ since all states are localized by disorder in one and two dimensions if the vector potential is real Localization in this specific kind of non-Hermitian quantum mechanics is referred to as "directed localization,", 3 because the imaginary vector potential breaks the symmetry between left-moving and right-moving particles, without breaking time-reversal symmetry

The analytical results of Ref 1 consist of expressions for the mobility edge and for the stretched-exponentral relaxation of delocalized states, and a solution of the onedimensional problem with a single impurity Here we go further, by solving the many-1mpurity case in one dimension Most of the technical results which we will need were derived previously in connection with the problem of localization in the presence of an imaginary scalar potential Physically, these two problems are entirely different an imaginary vector potential singles out a direction in space, while an imaginary scalar potential singles out a direction in time $A$ negative imaginary part of the scalar potential corresponds to absorption and a positive imaginary part to amplification One might surmise that amplification could cause a delocalization transition, but in fact all states remain localized in one dimension in the piesence of an imaginary scalar potential $^{67}$

Following Ref 1 we consider a disordered chain with the single-particle Hamiltonian

$$
\mathcal{H}=-\frac{w}{2} \sum_{J}\left(e^{h a} c_{j}^{\dagger}+c_{j}+e^{-h a} c_{j}^{\dagger} c_{J+1}\right)+\sum_{J} V_{J} c_{j}^{\dagger} c_{J}
$$

The operators $c_{j}^{\dagger}$ and $c_{j}$ are creation and annihilation operators, $a$ is the lattice constant, and $w$ the hopping parameter The random potential $V$, is chosen independently for each site, from a distribution with zero mean and variance $u^{2}$ For weak disorder (mean free path much larger than the wavelength), higher moments of the distribution of $V_{j}$ are not relevant The Hamiltonian is non-Hermitian because of the real parameter $h$, corresponding to the imaginary vector potential The chain of length $L$ is closed into a ring, and the problem is to determine the eigenvalues $\varepsilon$ of $\mathcal{H}$ If $\varepsilon$ is an ergenvalue of $\mathcal{H}$, then also $\varepsilon^{*}$ is one - because $\mathcal{H}$ is real Real $\varepsilon$ corresponds to localized states, while complex $\varepsilon$ corresponds to extended states

To solve this problem, we reformulate it in terms of the $2 \times 2$ transfer matrix $M_{h}(\varepsilon)$ of the chain, which relates wave amplitudes at both ends ${ }^{8}$ The energy $\varepsilon$ is an eigenvalue of $\mathcal{H}$ if and only if $M_{h}(\varepsilon)$ has an eigenvalue of 1 The use of the transfer matrix is advantageous, because the effect of the imaginary vector potential is just to multiply $M$ with a scalar,

$$
M_{h}(\varepsilon)=e^{h L} M_{0}(\varepsilon)
$$

The energy spectrum is therefore determined by

$$
\operatorname{det}\left[1-e^{h L} M_{0}(\varepsilon)\right]=0
$$

Time-reversal symmetry 1 mplies $\operatorname{det} M_{0}=1$ Hence the determinantal Eq (3) is equivalent to ${ }^{11}$

$$
\operatorname{tr} M_{0}(\varepsilon)=2 \cosh h L
$$

We seek the solution in the limit $L \rightarrow \infty$

Since $M_{0}$ is the transfer matrix in the absence of the imaginary vector potential $(h=0)$, we can use the results in the literature on localization in conventional onedimensional systems (having an Hermitian Hamiltonian) ${ }^{9}$ The four matrix elements of $M_{0}$ are given in terms of the reflection amplitudes $r, r^{\prime}$ and the transmission amplitude $t$ by

$$
\begin{gathered}
\left(M_{0}\right)_{11}=-(1 / t) \operatorname{det} S, \quad\left(M_{0}\right)_{12}=t^{\prime} / t, \\
\left(M_{0}\right)_{21}=-r / t, \quad\left(M_{0}\right)_{22}=1 / t,
\end{gathered}
$$

where $\operatorname{det} S=r r^{\prime}-t^{2}$ is the determinant of the scattering matrix (There is only a single transmission amplitude because of time-reversal symmetry, so that transmission from left to right is equivalent to transmission from right to left ) The transmission probability $T=|t|^{2}$ decays exponentially in the large- $L$ limit, with decay length $\xi$

$$
-\lim _{L \rightarrow \infty} L^{-1} \ln T=\xi^{-1}
$$


The energy dependence of $\xi$ is known for weak disorder, such that $|k| \xi \gg 1$, where the complex wave number $k$ is related to $\varepsilon$ by the dispersion relation

$$
\varepsilon=-w \cos k a \text {. }
$$

For real $k$, the decay length is the localization length $\xi_{0}$, given by ${ }^{10}$

$$
\xi_{0}=a(w / u)^{2} \sin ^{2}(\operatorname{Re} k a) .
$$

(Since $\xi_{0}$ is of the order of the mean free path $\mathscr{\ell}$, the condition of weak disorder requires $\ell$ large compared to the wavelength.) For complex $k$, the decay length is shorter than $\xi_{0}$, regardless of the sign of $\operatorname{Im} k$, according to ${ }^{6,7}$

$$
\xi^{-1}=\xi_{0}^{-1}+2|\operatorname{Im} k| \text {. }
$$

We use these results to simplify Eq. (4). Upon taking the logarithm of both sides of Eq. (4), dividing by $L$ and taking the limit $L \rightarrow \infty$, one finds

$$
|h|-\frac{1}{2} \xi^{-1}=\lim _{L \rightarrow \infty} L^{-1} \ln |1-\operatorname{det} S|,
$$

where we have used $L^{-1} \ln f \rightarrow L^{-1} \ln |f|$ as $L \rightarrow \infty$ for any complex function $f(L)$. For complex $k$, the absolute value of $\operatorname{det} S$ is either $<1$ (for $\operatorname{Im} k>0$ ) or $>1$ (for $\operatorname{Im} k<0$ ). As a consequence, $\ln |1-\operatorname{det} S|$ remains bounded for $L \rightarrow \infty$, so that the right-hand side of Eq. (10) vanishes. Substituting Eq. (9), we find that complex wave numbers $k$ satisfy

$$
|\operatorname{Im} k|=|h|-\frac{1}{2} \xi_{0}^{-1} .
$$

Together with the expression (8) for the localization length $\xi_{0}$, this is a relation between the real and imaginary parts of the wave number. Using the dispersion relation (7), and noticing that the condition $|k| \xi \gg 1$ for weak disorder implies $|\operatorname{Im} k| \ll|\operatorname{Re} k|$, we can transform Eq. (11) into a relation between the real and imaginary parts of the energy,

$$
|\operatorname{Im} \varepsilon|=|h| a \sqrt{w^{2}-(\operatorname{Re} \varepsilon)^{2}}-\frac{u^{2}}{2 \sqrt{w^{2}-(\operatorname{Re} \varepsilon)^{2}}} .
$$

The support of the density of states in the complex plane consists of the closed curve (12) plus two line segments on the real axis, ${ }^{12}$ extending from the band edge $\pm w$ to the mobility edge $\pm \varepsilon_{c}$. The real eigenvalues are identical to the eigenvalues at $h=0$, up to exponentially small corrections. The energy $\varepsilon_{c}$ is obtained by putting $\operatorname{Im} \varepsilon=0$ in Eq. (12), or equivalently be equating ${ }^{1} 2 \xi_{0}$ to $1 /|h|$, hence

$$
\varepsilon_{c}=\left(w^{2}-u^{2} / 2|h| a\right)^{1 / 2} .
$$

The delocalization transition at $\varepsilon_{\iota}$ exists for $|h|>h_{c}=\frac{1}{2} u^{2} / w^{2} a$.

In Fig. 1(a), the analytical theory is compared with a numerical diagonalization of the Hamiltonian (1). The numerical finite- $L$ results are consistent with the large- $L$ limit (dashed curve). To leading order in $1 / L$, fluctuations of $\operatorname{Im} \varepsilon$ around the large- $L$ limit (12) are governed by fluctuations of the transmission probability $T$. [Fluctuations of $L^{-1} \ln T$ are of order $L^{-1 / 2}$, while the other fluctuating contributions to Eq. (4) are of order $L^{-1}$.] The variance of $\ln T$ for large $L$ is known, ${ }^{13}$
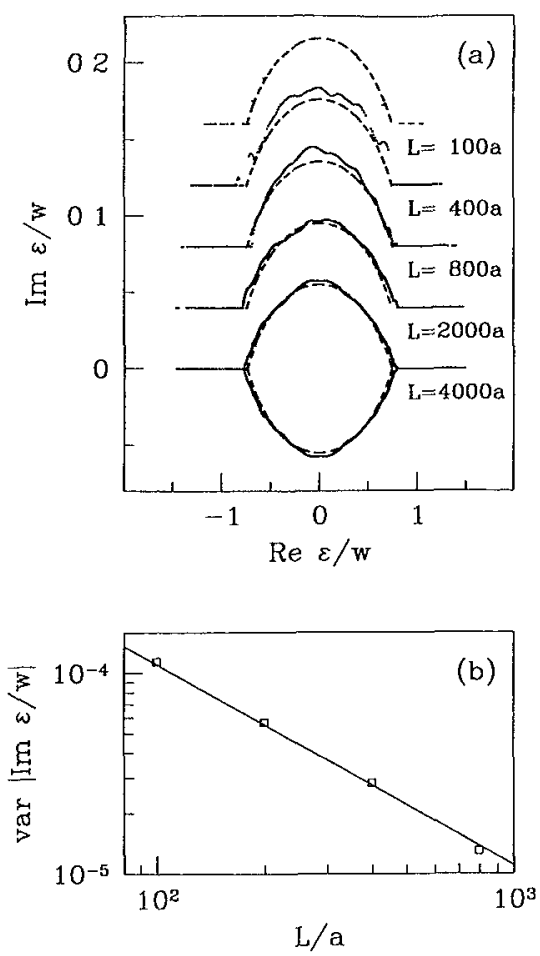

FIG. 1. (a) Data points: eigenvalues of the Hamiltonian (1), for parameter values $h a=0.1, u / w=0.3$, and for five values of $L / a$. Dashed curves: analytical large- $L$ limit, given by Eq. (12). (Except for the case $L=4000 a$, spectra are offset vertically and only eigenvalues with $\operatorname{Im} \varepsilon \geqslant 0$ are shown.) (b) Variance of the imaginary part of the eigenvalues as a function of the sample length, for $\operatorname{Re} \varepsilon \approx 0$ and for the same parameter values as in (a). The data points are the numerical results for 1000 samples. The solid line is the analytical result (15).

$$
\operatorname{var} \ln T=\frac{2 L}{\xi_{0}}+8 L|\operatorname{Im} k| e^{4 \xi_{0}|\operatorname{Im} k|} \operatorname{Ei}\left(-4 \xi_{0}|\operatorname{Im} k|\right),
$$

where $\mathrm{Ei}$ is the exponential integral. Equating $|\operatorname{Im} k|=|h|+\frac{1}{2} L^{-1} \ln T$, we find $\operatorname{var}|\operatorname{Im} k|=\frac{1}{4} L^{-2} \operatorname{var} \ln T$ and thus

$$
\operatorname{var}|\operatorname{Im} \varepsilon|=\frac{a^{2}}{2 L \xi_{0}}\left[1+2 \gamma e^{2 \gamma} \operatorname{Ei}(-2 \gamma)\right]\left[w^{2}-(\operatorname{Re} \varepsilon)^{2}\right]
$$

where $\gamma=2|h| \xi_{0}-1$. In Fig. 1(b) we see that Eq. (15) agrees well with the results of the numerical diagonalization. The fluctuations $\Delta \operatorname{Im} \varepsilon$ are correlated over a range $\Delta \operatorname{Re} \varepsilon$ which is large compared to $\Delta \operatorname{Im} \varepsilon$ itself, their ratio $\Delta \operatorname{Im} \varepsilon / \Delta \operatorname{Re} \varepsilon$ decreasing $\propto L^{-1 / 2}$. This explains why the complex eigenvalues for a specific sample appear to lie on a smooth curve [see Fig. 1(a)]. This curve is sample specific and fluctuates around the large- $L$ limit (12).

In conclusion, we have presented an analytical theory for the delocalization transition in a single-channel disordered wire with an imaginary vector potential. We find good agreement with numerical diagonalizations, both for the relation between the real and imaginary parts of the energy in the 